\title{
BMJ Open Protocol for a longitudinal qualitative study: survivors of childhood critical illness exploring long-term psychosocial well-being and needs - The SCETCH Project
}

\author{
Joseph C Manning, ${ }^{1,2}$ Pippa Hemingway, ${ }^{1}$ Sarah A Redsell ${ }^{3}$
}

To cite: Manning JC, Hemingway P, Redsell SA. Protocol for a longitudinal qualitative study: survivors of childhood critical illness exploring long-term psychosocial well-being and needs-The SCETCH Project. BMJ Open 2014;4:e004230. doi:10.1136/bmjopen-2013004230

- Prepublication history for this paper is available online. To view these files please visit the journal online (http://dx.doi.org/10.1136/ bmjopen-2013-004230).

Received 11 October 2013 Revised 19 November 2013 Accepted 20 November 2013

CrossMark

\section{${ }^{1}$ School of Health Sciences, Faculty of Medicine and Health Sciences, University of Nottingham, Nottingham, UK ${ }^{2}$ Paediatric Intensive Care Unit, Nottingham Children's Hospital, Nottingham University Hospitals NHS Trust, Nottingham, UK ${ }^{3}$ Faculty of Health, Social Care and Education, Anglia Ruskin University, Cambridge, UK}

\section{Correspondence to} Joseph C Manning; joseph. manning@nottingham.ac.uk

\section{ABSTRACT}

Introduction: Life-threatening critical illness affects over a quarter of a million children and adolescents ( $0-18$ years old) annually in the USA and the UK. Death from critical illness is rare; however, survivors and their families can be exposed to a complex array of negative physical, psychological and social problems. Currently, within the literature, there is a distinct paucity of child and adolescent survivor self-reports, thus limiting our understanding of how survivors perceive this adversity and subsequently cope and grow in the long-term following their critical illness. This study aims to explore and understand psychosocial well-being and needs of critical illness survivors, 6-20 months post paediatric intensive care admission.

Methods and analysis: A longitudinal, qualitative approach will provide a platform for a holistic and contextualised exploration of outcomes and mechanisms at an individual level. Up to 80 participants, including 20 childhood critical illness survivors and 60 associated family members or health professionals/teachers, will be recruited. Three interviews, 7-9 weeks apart, will be conducted with critical illness survivors, allowing for the exploration of psychosocial well-being over time. A single interview will be conducted with the other participants enabling the exploration of contextual information and how psychosocial well-being may inter-relate between critical illness survivors and themselves. A 'tool box' of qualitative methods (semi-structured interviews, draw and tell, photo-elicitation, graphic-elicitation) will be used to collect data. Narrative analysis and pattern matching will be used to identify emergent themes across participants.

Ethics and dissemination: This study will provide an insight and understanding of participants' experiences and perspectives of surviving critical illness in the long term with specific relation to their psychosocial wellbeing. Multiple methods will be used to ensure that the findings are effectively disseminated to service users, clinicians, policy and academic audiences. The study has full ethical approval from the East Midlands Research Ethics Committee and has received National Health Service (NHS) governance clearance.

\section{Strengths and limitations of this study}

Methodological approach will provide new insights and greater breadth of understanding into phenomena.

- Creative qualitative data collection methods will provide a platform for experiences and perceptions of well-being to be represented from previously unheard populations.

- Longitudinal design has potential for high attrition that could limit the sample size.

\section{INTRODUCTION}

Acute life-threatening critical illness in childhood can be caused by a wide variety of diseases and injuries. ${ }^{1}$ However, irrespective of cause, without prompt or appropriate treatment, a rapid loss of physiological capacity may be experienced which can result in significant morbidity or death. Immediate specialist interventions are therefore required to support and treat critically ill children and adolescents with continued management involving intensive monitoring and invasive treatments. ${ }^{2}$

More than one-quarter of a million children and adolescents annually in the USA and the UK require admission to paediatric intensive care unit (PICU). ${ }^{3}{ }^{4}$ Over the past four decades, there have been significant advances in paediatric critical care which has led to lower mortality from childhood critical illness $(<4 \%) .{ }^{4}$ With regard to the majority of children and adolescents who survive critical illness, residual physical, psychological and social impact has been purported to manifest. ${ }^{5-7}$

Impact of critical illness on children and adolescents

During their PICU admission, critically ill children and adolescents are exposed to a 
significant number of invasive procedures, ${ }^{8-10}$ reported to be, on an average, seven per day (89 per total stay). ${ }^{11}$ Such procedures include intubation and mechanical ventilation, endotracheal suctioning, intravenous cannulation and central line placement, chest tube insertion and urinary catheter placement. In addition, critically ill patients may receive numerous infusions of medications which can have disturbing side effects such as hallucinations and delirium. ${ }^{12}$ The PICU environment is also reported to be noisy and frightening due to large numbers of staff (doctors, nurses and allied health professionals) as well as monitoring equipment and machinery. Noise levels are reported as much as eight times higher than recommended levels by the WHO. ${ }^{13}$

Consequently, the time spent unwell in PICU has been reported to result in a range of psychological manifestations in child and adolescent survivors that can include negative thoughts and emotions, decreased levels of selfesteem and reduced perceptions of control. Nightmares and hallucinations have been reported to manifest for a number of months post-PICU discharge. ${ }^{10}{ }^{14} \mathrm{Up}$ to $5 \%$ of survivors discharged from PICU are reported to have delirium, with older children having a higher prevalence than younger children. ${ }^{8}$ Distressing thoughts and delusional memories have also been purported to occur within 2 months from discharge. ${ }^{15}$ Negative memories that relate to traumatic aspects of the child's admission include the PICU environment, pain, interventions and equipment. However, prevalence of negative thoughts varies from $15 \%$ to $43 \%,{ }^{10}$ which could be attributed to differences in timing of post discharge data collection or methods used for obtaining and categorising the types of memories.

Fears and anxieties have been purported to manifest up to 6 months after PICU care. ${ }^{9-11}{ }^{15-17}$ However, there is a considerable variation in the prevalence of increased levels of fear, ranging from $13 \%{ }^{11} 17$ to $50 \% .^{10}$ Negative emotions have been reported to emanate from perceived dangers of being on PICU, which include being unable to eat or move, medical procedures and the expectation of pain, ${ }^{18}$ separation from parents and the death of other children. ${ }^{11}$ Children exposed to higher numbers of invasive procedures and younger children have been reported to have greater levels of fear post discharge. ${ }^{19}$

Self-esteem and perceived level of control have also been reported to be negatively affected. Critical illness has been reported to involve a qualitatively diverse experience, which renders the child or adolescent unable to 'rally in the adversity' that it is exposed to. ${ }^{14}$ Child survivors are reported to experience changes in relation to their behaviour, memory, attention span, selfesteem and self-confidence. ${ }^{11} 14{ }^{16}$ Elevated levels of avoidance, low self-esteem and depression have been reported in PICU survivors 1-month post discharge ${ }^{16}$ with younger children and those more severely ill having lower perceived levels of control $^{11}$ up to 6 months post discharge.
Stress and post-traumatic stress disorder (PTSD) in children and adolescents following PICU care has been widely investigated and reported to occur up to 12 months post admission. ${ }^{6} 91215$ 20-23 The prevalence of PTSD symptoms in PICU survivors ranges from $23 \%^{9} 15172124$ to $34 \%^{22}$; however, research conflicts with regard to the trajectory of prevalence over time. ${ }^{17}{ }^{21}{ }^{23}$ Factors associated with increased levels of stress and PTSD symptoms include invasive procedures ${ }^{17}$ and illness severity. ${ }^{22}{ }^{25}$ Child age has also been reported as a significant predictor of long-term PTSD, with younger children having higher number of symptoms than older children. ${ }^{26}$ However, there is also some evidence to suggest that there is no association between level of PTSD symptoms and age, gender or length of stay. ${ }^{9}$

Quality of life (QoL) of child and adolescent survivors has also been purported to be negatively affected up to 6 years post-PICU discharge. Increased severity of illness and prolonged length of PICU stay have been associated with poor QoL. ${ }^{27}$ In addition, poor neurological outcome has been associated with either poor or fair QoL (as measured on the Royal Alexandra Hospital for Children Measure of Function).$^{28}$ However, in contrast, children who experience trauma, cardiac or respiratory-related illnesses have been reported to have better QoL scores than those children with malignancies (cancers). ${ }^{27}$ Younger children have been reported to have better QoL scores when compared with older children who have survived a critical illness. ${ }^{27} 29$

Socially, child and adolescent survivors have been reported to experience increased time off school, ${ }^{14}{ }^{21}$ bullying, ${ }^{14}$ and many outpatient and inpatient visits. ${ }^{14}{ }^{21}$ The social functioning domain of QoL has been reported to be negatively affected at 3 months post discharge. ${ }^{29}$

\section{Impact of critical illness on the family}

Spending time unwell in PICU can be a period of crisis for a family, ${ }^{30}$ inciting considerable levels of fear and anxiety with regard to any potential losses that may be faced (ie, brain damage, disfigurement or death). ${ }^{12}$ Parents have been reported to experience high levels of anxiety and PTSD symptoms post-PICU, with a higher prevalence reported in mothers compared with fathers. ${ }^{5}$ Parental stress levels have been reported to increase when there is uncertainty over the child's prognosis or when there are increased demands of having a child with a chronic illness or complex needs. ${ }^{12}$ A direct relationship between illness severity and family social impact has also been reported in some families, with a negative impact on marital and domestic partner relationships being described. ${ }^{5}$ However, some studies do purport positive impact on the family with the survival of severe critical illnesses (eg, near-death events) resulting in stronger family-child relationships. ${ }^{5}$ Collectively, positive and negative outcomes of the experience have been documented for families of these children. ${ }^{5}$ 
Limitations of the existing literature

Despite a growing body of literature in this field, a number of limitations exist that have impeded a comprehensive understanding of the overall experiences of children and adolescents who survive PICU. ${ }^{6}$

UK audit data identifies that around $70 \%$ of admissions to PICU are children aged 3 years and under, ${ }^{4}$ which indirectly infers that the majority of survivors do not have the cognitive developmental capability to selfreport (eg, those who are preverbal or who are in their preoperational stage ${ }^{31}$ ). This could contribute to explaining why adult voices appear to exclusively report experience or impact on behalf of childhood survivors. ${ }^{28}{ }^{32-34}$ However, adult voices continue to dominate reports for those children and adolescents with the cognitive development capabilities. Moreover, researchers have imposed specific methods, such as structured interviews and questionnaires ${ }^{11213536}$ or single one-off timepoints to collect information (such as 3 and 9 months postcritical illness), ${ }^{22}{ }^{29}$ where the specific relevance to the child or adolescent survivor is unclear. Collectively, this has resulted in the survivors' long-term experiences, views, descriptions, meanings and needs being predominantly lost, neglected or in some cases negated within the literature.

Problematising the impact and experience of surviving childhood critical illness has been the dominant approach used in the existing studies, resulting in a body of literature that is focused on negative aspects of survival. Subsequently, deductive claims have been made in relation to the causal inference of associated variables, such as anxieties, PTSD symptoms or poor QoL without exploration of contextual factors or events prior to critical illness that may have been influential. Collectively, this has impeded understanding of the potential complexity of psychological and social wellbeing of childhood survivors of critical illness. Moreover, until recently, ${ }^{30}$ this has hindered the development of theoretical models that recognise the significance and interplay of the survivor within their social world.

This stance is confirmed through the findings of a systematic review of qualitative studies in this field conducted by the authors. ${ }^{37} 38$ The findings concluded that the long-term psychological and social well-being trajectory of surviving critical illness in childhood remains largely unclear. The current understanding of how these children and adolescents cope and grow in relation to their psychological, social and emotional well-being is absent from the literature. ${ }^{6}$ Thematic synthesis does provide an insight into the potential multifaceted and complex transformations that children and adolescents can encounter. However, it is unclear whether the outcomes are isolated to specific times, or they are present throughout the experience of survival, or whether and how they may change over time. ${ }^{38}$ In addition, it is also uncertain whether there is an interplay between the reports and perceptions of significant others in survivors' lives (eg, parents, siblings or teachers) and whether or how these may influence survivors' well-being.

Clearly, further exploratory empirical research is required in order to gain a better understanding of this phenomenon.

\section{METHODS AND ANALYSIS}

\section{Study aim}

The aim of this study was to explore and understand psychological and social well-being, in the long term, as perceived and described by children and adolescents who have survived an acute life-threatening critical illness.

\section{Research questions}

How is psychological and social well-being described, perceived and experienced by childhood survivors at 6-20 months after an acute life-threatening critical illness?

a. How is psychological and social well-being described by child and adolescent survivors and how does this manifest over time?

b. How do children and adolescents perceive any changes to their psychological and social well-being?

c. How do children and adolescents' meanings and descriptions of psychosocial well-being compare with those of important others within their lives?

d. How do children and adolescents respond to any changes in psychological and social well-being following an acute life-threatening critical illness?

e. What are the long-term psychological and social needs of children and adolescents who survive acute life-threatening critical illness?

\section{Study design}

This is a prospective, longitudinal qualitative study. We propose that this approach will allow for a rich, in-depth and holistic investigation into context, mechanisms and outcomes. ${ }^{39}{ }^{40}$ The longitudinal collection of multiple sources of data will provide contextualised, converging and emerging lines of inquiry, ${ }^{41}$ allowing for interrelations between critical illness survivors and their families psychosocial well-being to be explored.

\section{Sample and recruitment}

\section{Setting}

The research setting is a large University Hospital NHS Trust in England that provides up to level four paediatric intensive care services ${ }^{2}$ to critically ill $0-18$ year-olds from the geographical catchment of the East Midlands. The PICU has approximately 350 admissions per annum ${ }^{42}$ and is situated in a large tertiary children's hospital that provides a number of regional and supra-regional specialist services (paediatric trauma; paediatric neurosurgery; paediatric oncology; nephrology; cleft lip and palate). Children and adolescents who require critical care due to primary cardiac or hepatic conditions are referred to other centres for specialist care. 


\section{Sampling}

Childhood critical illness survivors will be identified and recruited through a sequential sampling strategy that incorporates theoretical and snowball sampling. Theoretical sampling is defined by Emerson (ref. ${ }^{43}$, p.360) as sampling, '...in which new observations are selected to pursue analytically relevant distinctions rather than establish the frequency or distribution of phenomena'. In addition to eligibility criteria (shown in box 1), a theoretical sampling framework will also be used to ensure variety of participants. This framework will be based on the two variables: reason for admission to PICU (presenting clinical condition) and time since PICU admission. To gain a maximum variation, the sampling framework will seek two eligible participants from each diagnosis group, as defined by the International Classification of Diseases-10, ${ }^{44}$ for inclusion in the study. Disease groups included circulatory system, digestive system, genitourinary system, haematological, infections, injury/poisonings, metabolic disorders, neoplasms, neurological and respiratory system.

The study will also recruit other participants (such as family members of the child or adolescent). These will be selected on the basis of whether the child or adolescent survivor perceives them to be an important person in their life. Therefore, these are an undefined population and unknown to the researchers from the outset. Snowball sampling has been advocated for sampling hidden populations ${ }^{45}{ }^{46}$ and will be utilised. This approach collects a sample referred or nominated by the existing study participants, hence the term 'chainreferral method' was ascribed to this approach. The way in which this sampling technique is operationalised in studies varies within the literature according to the population and topic being studied. ${ }^{45} 46$ Therefore, each child or adolescent survivor will identify and invite other people to participate in the study. This is deemed important for two key reasons. First, it confirms the collaborative ethos that underpins this study in attempting to harness the child/adolescents' voice and recognises

Box 1 Eligibility criteria for the selection of childhood critical illness survivors

\section{Inclusion criteria}

1. Children and adolescents aged between 6 and 18 years

2. Survived unplanned admission to paediatric intensive care unit (PICU)

3. Invasively intubated and ventilated

4. Three to 12 months since discharge from PICU

5. Consents/assents to participate in the study

Exclusion criteria

1. Unable to fulfil the inclusion criteria

2. Critical illness due to child protection issues/non-accidental injuries

3. Children and adolescents who have survived critical illness but are on an active end of life care pathway

4. Non-English speaking. their agency in the research. Second, only the child/ adolescent can identify who they feel are significant people in their life. Figure 1 illustrates a schematic diagram of the study design.

\section{Sample size justification}

The purpose of exploratory qualitative research is to gain depth and understanding, and therefore the sample size should reflect this. ${ }^{47}$ Morse ${ }^{48}$ suggests that the greater the useable data elicited, the fewer participants are needed. This study proposes to collect a large amount of different types of data (audio and visual data), from a number of sources (eg, child and adolescent survivors, their family, teachers and health professionals), and at different time points (1, 3 and 6 months from consenting). Therefore, a maximum of 80 participants will be recruited consisting of 20 child/adolescent critical illness survivors and 60 significant other people (such as parents, siblings, health professionals and teachers).

\section{Data collection}

The potential participants in this study may vary in chronological age, developmental, cognitive and physical ability. Therefore, we propose to use a range of familiar and interesting qualitative data collection methods in order to engage with this potentially diverse sample. The use of multiple techniques in research exploring the lives of children and adolescents is advocated within the literature as it provides flexibility by allowing children/adolescents to communicate their experiences, ideas and feelings in their preferred way, ${ }^{41}{ }^{49}$ thus upholding a child and adolescent-centred ethos.

Data collection methods were selected from studies that have effectively used participatory or collaborative approaches to research with children and adolescents. Two predominant groups of techniques were identified from the literature and will make up the 'tool box of methods' that will be used in this study: talking, conversations and interviews ${ }^{50-52}$; and art-based techniques such as drawings, paintings, photography and collage. ${ }^{53-56}$

\section{Semi-structured interviews}

Face-to-face semi-structured interviews will be used as the primary method of data collection to allow the participants to communicate their experiences, feelings and emotions. Despite concerns apparent in the literature with regard to the power dynamics of undertaking interviews with children and adolescents, ${ }^{57}$ there continues to be prolific and effective use in child health research. ${ }^{50-52}$ 58-60

During the interviews, the researcher will actively encourage participants to freely construct their responses and narratives. However, in order to facilitate an in-depth exploration of abstract topics such as thoughts, feelings and meanings, an interview topic schedule will be used (with sensitising concepts elicited from the literature). These topics will not be structured 


\section{Screening and theoretical sampling of eligible patients}

Retrospective review of local admission database using eligibility criteria. Eligible patients status (dead/alive) and postal address checked on patient admission system. If more than 20 eligible participants are identified then participants selected according to presenting clinical condition to PICU (two patients from each diagnosis group as defined by the ICD-10).

\section{$\downarrow$}

\section{Approach eligible patients $(\mathrm{n}=\mathbf{2 0})$}

Standardised study invitation pack (letter with response slip, stamped addressed envelope, and participant information sheets) sent to eligible participants via postal mail by PICU team. If no response after 14 days, reminder letter sent by clinical team.

Informed consent and assent $(\mathrm{n}=\mathbf{2 0})$

A face to face information visit was conducted by the researcher with respondents (e.g. child/adolescent survivor and parent/legal guardian). This allowed for additional information to be provided, any questions to be answered, and informed consent and assent (if $<16$ years old) to be obtained. Identify other people to invite to participate in the study (maximum $n=3$ ).

\section{Data collection visit 1}

Conducted as soon as possible after consenting.

- Semi-structured interview including photoelicitation and draw and tell

- $\quad+$ /-graphic-elicitation activity-self portrait

- +/-graphic elicitation activity-time lining (past)

On-going collection of data ( $1-3$ months)

- Photographs/drawings/ artefacts

- Writing

\section{Data collection visit 2}

3 months after consenting

- Semi-structured interview including photoelicitation and draw and tell

- $\quad+$ /-graphic-elicitation activity-self portrait

- +/-graphic elicitation activity-time lining (past)

\section{On-going collection of data (3-6 months)}

- Photographs / drawings / artefacts

- Writing

$\downarrow$

\section{Data collection visit 3}

6 months after consenting

- Semi-structured interview including photoelicitation and draw and tell

- $\quad+$ /-graphic-elicitation activity-self portrait

- +/-graphic elicitation activity- time lining (future)

- Debrief conversation

Figure 1 Schematic diagram of study design. 
questions but prompts that allow the researcher to tailor the language, phrases and syntax to the cognitive and developmental ability of the participant as much as possible. Owing to the iterative and inductive nature of this study, ${ }^{39}$ preliminary data analysis will be undertaken after each data collection visit to allow for any emerging issues or questions to be explored and interview schedules to be modified.

\section{Art-based techniques}

Art-based techniques, such as artwork, drawings and photography, have been extensively advocated for use in research with children and adolescents. ${ }^{55} 6162$ However, these techniques are underused in health-related research. ${ }^{56}$ Art-based techniques are suggested to act as a symbolic language, ${ }^{61}{ }^{62}$ which are a 'powerful medium' for collecting the experiences, views and perceptions of children and adolescents from 'a wide range of the developmental continuum, ${ }^{55}$

It is recognised that some children and adolescents can have difficulty in verbally articulating sensitive issues, fears and feelings. ${ }^{63}$ In addition, participants in this study may vary according to cognitive ability and developmental level. Therefore, art-based techniques will be used to facilitate the participant to communicate through a fun medium ${ }^{64}{ }^{65}$ that is suitable for all children, irrespective of age and stage of cognitive development.

\section{Drawings and graphic-elicitation}

Drawings have been used as an open-ended approach to elicit child and adolescents voices. ${ }^{57} 60$ 66-68 A combination of 'impromptu' (created through prompt by the researcher) and 'spontaneous' (created without direction) approaches to drawings ${ }^{56}$ will be utilised, which will be governed by the context, developmental level and ability of the child/adolescent. Established graphic-elicitation techniques that explore psychological and social identities (such as self-portrait and time-lining activities $^{5369}$ ) will be utilised.

\section{Photography and photo-elicitation}

Photographs have been reported to allow the researcher to view the world through the participants' eyes ${ }^{55}$ and provide in-depth knowledge into a moment in time. ${ }^{70}$ Similar to the use of drawings, photographs can also be used to discuss, evoke and explore participants' feelings, experiences and views ${ }^{55} 5671$ and enables the researcher to bridge the 'psychological and physical realities'. ${ }^{72}$ Participants will have the option to choose to take or use photographs which will act as a platform for discussion during the interviews.

\section{Artefacts}

Artefacts that may facilitate the child/adolescent to communicate their experiences, views and needs will also be used to aid data collection (ie, a toy, game or a patient's name band). The use of artefacts and props has been advocated for use in research with children and include masks, puppets or dolls. It is suggested that a major benefit of this technique is that it allows participants to be slightly removed from their stories/explanations which is useful when articulating potentially emotionally laden events. ${ }^{56}$

\section{Study procedures}

Following consent being obtained, the experiences of each child/adolescent survivor will be explored longitudinally, with data being collected for a total of 6 months (see figure 1).

Data collection with child and adolescent survivors will involve a minimum of three face-to-face visits with the researcher. The researchers will provide each child/adolescent with a pack of stationery (paper, felt tips, pens and folder) and a digital camera to use for the duration of the study and will be encouraged to continue to collect data (photographs/drawings) in between visits with the researcher. At the beginning of each visit, the researcher will reconfirm the child's/adolescent's willingness to participate in the study and restate that continued involvement is entirely voluntarily and they can withdraw at any time. Other participants will receive a single interview with the researcher.

For participant convenience, all study participants will be able to choose the date, time and location of the interviews. For those children and adolescents in fulltime education or employment, it is anticipated that the visits will occur in their own home, during evenings or at weekends. Participants will also be given the choice as to where the visits are located within the home (eg, lounge) and who else is present (eg, siblings).

With the participant's agreement, interviews will be audio-recorded using a digital voice recorder. As advocated by Truesdell, ${ }^{73}$ prior to the interview, the equipment will be checked to ensure whether it is functioning appropriately, allowing the researcher to concentrate on the participants' dialogue and engage in active listening.

\section{Data analysis}

All interviews will be audio-recorded and transcribed verbatim. The visual data (eg. photographs and drawings) will be inserted into the transcripts at the time-point discussed during the interview. All data will then be imported into a qualitative data analysis package (NVivo V.10), which will assist in managing, sorting and coding the data. Data analysis will be primarily conducted by JCM, with the other researchers (SAR and $\mathrm{PH}$ ) verifying the findings for consistencies and discrepancies in order to maximise the validity and reliability. Standard procedures for evaluating and ensuring rigour in qualitative research, as described by Mays and Pope ${ }^{74}$ and Seale, ${ }^{75}$ will be employed.

Data analysis will involve two broad stages: (1) narrative analysis and (2) pattern matching and synthesis. 


\section{Stage 1: narrative analysis}

The first stage will involve analysing the data from each participant. In order to maintain the timing and situationality of the data, as well as the significance and meaning of participants' voices, a narrative method of analysis will be used. ${ }^{76}$ The narrative psychological analysis approach devised by McAdams ${ }^{77}$ and subsequently developed by Crossley ${ }^{78}$ will be applied. This approach involves five steps and is based on the assumption that an insight into an individual's psychological and social realities can be gained through understanding the content and complexity of meanings produced in interview situations (ref. ${ }^{78}$, p. 88 ).

- Step 1: Reading and familiarising. Also referred to 'naïve reading', this stage will involve repeatedly reading through the whole transcript (about 5 or 6 times) in order to familiarise oneself with the material, enabling a general gist of emerging and significant themes. ${ }^{78}$ This process will allow the researchers to immerse themselves in the content and take note of the atmosphere of the interview.

- Step 2: Identifying important concepts to look for. This step involves establishing the principal elements of the 'personal narrative'. Narrative analysis takes note of the detail within the text, as well as emerging themes. While much of this attention to detail has been developed through conversation analysis (the pauses and silences), the specific use of language will be equally as important. ${ }^{78}$

- Step 3: Identifying 'narrative tone'. McAdams ${ }^{77} 79$ identifies narrative tone as the most pervasive feature of biographical narratives. Tone is conveyed in the content and the form of narratives. ${ }^{78}$ Therefore, this step focuses on what makes up the story (eg, events) and how these are portrayed (eg, optimism/sorrow/ despair).

- Step 4: Identifying 'imagery' and 'themes'. This step will involve identifying imagery (such as metaphors, descriptions and actual images) and dominant themes that demonstrate the significance and mechanisms that surround important events reported in the narrative. These will be mapped in relation to six aspects of the narrative: life chapters, key events, significant people, future script, current problems and personal ideology. ${ }^{78}$

- Step 5: Weaving it all together. The final step in the narrative analysis will involve reintegrating all the components of the analysis together into a coherent story.

\section{Stage 2: pattern matching and synthesis}

A pattern matching analytical approach will be applied. ${ }^{39}$ Themes identified from the narrative analysis (stage 1) will be explored and compared between participants, specifically looking for patterns, similarities and differences. In addition 'word tables ${ }^{39}$ will be used to collate and present emergent characteristics, issues or themes through the aggregation of occurrences.
Collectively, this stage will provide a detailed description and understanding of over-riding themes ${ }^{39}$ focusing on psychosocial well-being and needs.

\section{ETHICS AND DISSEMINATION}

\section{Patient and public involvement}

To uphold the collaborative ethos of this study, and to ensure relevance and quality of the research, ${ }^{80-82}$ involvement was sought from children, adolescents and their families when developing the study design. Seven children and adolescents (aged 6-15 years) who had varying experiences of healthcare (eg, chronically unwell, healthy) and three parents (2 mothers, 1 father) volunteered to provide guidance and advice. In line with guidance ${ }^{83}$ volunteers received a gift voucher $(£ 5.00)$ as a token of appreciation for their involvement.

Collectively, recommendations were made to the recruitment process, data collection methods and participant information. Positive feedback was elicited from all children and adolescents in relation to the methods used to collect data, with flexibility and choice being fundamental. However, detailed feedback on the study information revealed a number of issues that related to highlighting ambiguous or confusing statements; font size and document length (eg, too small and too long); requesting changes for more accessible terminology (eg, change 'explain' to 'talk about things') and appealing for the documents to be more colourful. Feedback was collated and the protocol and study information were revised to reflect these changes.

\section{Informed consent}

Informed consent will be obtained through a face-to-face visit between the researcher (JCM) and eligible participant (including their parent/legal guardian if $<16$ years old). At this visit, the researcher will explain further the details of the study, answering any questions that the participant and parent/legal guardian may have concerning taking part. For participants aged under 16 years, written consent will be gained from the parent or legal guardian as well as assent from the child/adolescent. In the event of any conflict between the parent and child, the child will not participate in the study. As this is a longitudinal study, requiring data collection at a number of time points over a 6-month period, the researchers will reconfirm willingness to continue in the study from the child or adolescent at each visit. Participants will be able to withdraw from the study at any time. In the event of their withdrawal, as data collected so far cannot be erased, the researchers will seek written consent to use the data in the final analyses.

\section{Ethical considerations}

It is reported extensively in the literature that conducting research with children and adolescents generates a multitude of ethical challenges that must be identified and addressed. ${ }^{84-88}$ Four main areas were identified as 
specific areas for consideration when developing the protocol and include: (1) power dynamics; (2) researching potentially sensitive and emotive topics; (3) safeguarding and (4) maintaining professional boundaries.

1. Power dynamics: It is recognised that managing the power relationships between children/adolescents and adults during data collection can pose a particular challenge. ${ }^{84}$ Threats to participant researcher parity are particularly problematic when utilising an interview method ${ }^{89}$ as the interviewer could maintain control and power by leading the participant in the type and way he/she responds to the questions. Therefore, attempts will be made to minimise/eliminate this by embedding the research with a collaborative ethos that aims to empower the participant to make choices and decisions with regard to parts of the research process. ${ }^{62} 90$ For example, the child/ adolescent will choose what art-based techniques they would like to use (if any). In addition, the child/adolescent will remain the owner of any data generated and will control when and what (if anything) they share with the researcher.

2. Researching potentially sensitive and emotive topics: It must be recognised that the study participants may already have psychological sequalae (eg, post-traumatic stress symptoms) as a result of the critical illness experience. In addition, the information discussed during the data collection phase might be emotive or upsetting to participants and/or family members. Considering this, a number of mechanisms have been integrated into the study to address this potential issue. First, each child/adolescent survivor will be explored in-depth, which in itself may provide opportunity for issues, feelings and emotions to be discussed. This will be facilitated by data collection techniques that are sensitive to exploring potentially traumatic events, in a constructive and timely manner. Second, the study steering group will have the expertise and guidance of a paediatric clinical psychologist and therefore any issues identified can be referred to the steering group for discussion. This will allow the researcher to be guided with regard to signposting the participant to appropriate services or support. During data collection, if the participant becomes visibly upset, the researcher will: ask the parent/legal guardian to console the child/adolescent (if appropriate); offer to temporarily stop or terminate the visit; respect the decision made by the participant to stop/carry on the interview.

3. Safeguarding: To ensure the safety of the participants and the researchers, all visits with children and adolescents (<16-year-olds) will be conducted with the parent/legal guardian present. In situations where it is not possible or the child specifically requests for the parent(s) not to be present, a second researcher from the study team will be present. All study researchers have an enhanced Criminal Records Bureau (CRB) check and are registered health professionals bound by a code of professional conduct. ${ }^{91}$ Therefore, if at any time during data collection, a participant discloses information that relates to safeguarding or child protection this may have to be shared with other agencies (ie, social services).

4. Maintaining professional boundaries: Owing to the in-depth and exploratory nature of this qualitative research study and the longitudinal period for data collection, relationships may be formed between the participants and the researcher. To ensure that professional relationships are maintained between participants and the researcher, the researcher (JCM) will receive a minimum of $4 \mathrm{~h}$ of clinical supervision with a consultant paediatric clinical psychologist during the data collection period. This will allow for the researcher to reflect and learn from their experiences, practices and progress in the research. Participant's confidentiality and anonymity will be maintained during clinical supervision. It is also recognised that a certain level of dependency may develop between the participant and the researcher over the study period. In order to support the participants, at the final data collection visit, debriefing will be conducted. Owing to the inductive and exploratory nature of this study, it is not possible to predict all potential themes that may be discussed as this will be tailored to each participant. However, broad topics that might be discussed include: emphasising clearly that this is the end of the study and is the last visit; any issues/anxieties that they or their parent/ legal guardian might have about the study ending; signposting the participant, parent or legal guardian to other services or support groups; any feedback for the researcher on what it has been like participating in this study.

\section{Methods of dissemination of findings}

This paper serves as an important step in the dissemination of the findings by outlining the project background, providing a detailed description of methods and procedures and discussing a number of practical challenges that may be faced and how the study team will address these.

We propose that the findings from this study will contribute to addressing the significant gaps in the literature by providing, for the first time, a holistic, longitudinal, qualitative exploration into psychosocial well-being of surviving childhood critical illness. This study will also identify any outstanding needs that survivors and their families may have. Collectively, the findings from this study will act as the first stage in developing and informing any potential interventions (as outlined by Medical Research Council framework ${ }^{92}$ ) to support psychosocial well-being in this patient population.

We aim to disseminate the research findings to regional, national and international audiences including 
service users, clinicians, academics, service commissioners and policymakers. A local research dissemination and stakeholder event (bringing together service users, health professionals, managers and commissioners) will be held to identify best ways in which findings can be translated into current or new services. In addition, we will continue to work with our service users (children, adolescents and their families) to develop appropriate interventions, determined by the findings from the study. Dissemination will also include presentations at relevant research conferences, and we will publish papers in open access, high-quality, peer-reviewed journals.

Acknowledgements The authors would like to acknowledge and thank the children, adolescents and their parents who provided comments and feedback in relation to the design and participant materials for this study. The study team would also like to thank the study steering group. In particular, the authors would like to acknowledge the contribution of Dr Simona Lampariello (local collaborator for the study) and Dr Emily Talbot (member of the study steering group) for their comments on this protocol.

Contributors JCM, SAR and PH were responsible for the development and refinement of the protocol. The study is being conducted as part of JCM's $\mathrm{PhD}$ research. JCM will conduct all data collection and analysis under the supervision of SAR and PH. JCM wrote the initial draft of the manuscript. JCM, SAR and PH contributed to edited, and approved the final manuscript.

Funding This study is funded through a Translational Research Fellowship awarded to JCM from the National Institute for Health Research (NIHR) and Nottingham University Hospitals NHS Trust through the University of Nottingham.

\section{Competing interests None.}

Ethics approval The study will be conducted in accordance with the ethical principles that have their origin in the Declaration of Helsinki, 1996; the principles of Good Clinical Practice, and the Department of Health Research Governance Framework for Health and Social care, 2005. Full ethical approval has been received from East Midlands Research Ethics Committee (REC ref: Derby 1,12/EM/0230), along with research governance clearance from the appropriate National Health Service (NHS) Trust.

Provenance and peer review Not commissioned; externally peer reviewed.

Open Access This is an Open Access article distributed in accordance with the Creative Commons Attribution Non Commercial (CC BY-NC 3.0) license, which permits others to distribute, remix, adapt, build upon this work noncommercially, and license their derivative works on different terms, provided the original work is properly cited and the use is non-commercial. See: http:// creativecommons.org/licenses/by-nc/3.0/

\section{REFERENCES}

1. Advanced Life Support Group. Advanced paediatric life support: the practical approach. 5th edn. Oxford: BMJ Publishing Group (Wiley-Blackwell), 2011.

2. Paediatric Intensive Care Society. Standards for the care of critically ill children. 4th edn. London: PICS UK, 2010.

3. Odetola FO, Clark SJ, Freed GL, et al. A national survey of pediatric critical care resources in the United States. Pediatrics 2005;115: e382-6.

4. Paediatric Intensive Care Audit Network. Annual Report 2010-2013: Universities of Leeds and Leicester, 2013.

5. Shudy M, de Almeida ML, Ly S, et al. Impact of pediatric critical illness and injury on families: a systematic literature review. Pediatrics 2006;118(Suppl 3):S203-18.

6. Rennick JE, Rashotte J. Psychological outcomes in children following pediatric intensive care unit hospitalization: a systematic review of the research. J Child Health Care 2009;13:128-49.

7. Nelson LP, Gold Jl. Posttraumatic stress disorder in children and their parents following admission to the pediatric intensive care unit: a review. Pediatr Crit Care Med 2012;13:338-47.
8. Schieveld JNM, Leroy PLJM, van Os J, et al. Pediatric delirium in critical illness: phenomenology, clinical correlates and treatment response in 40 cases in the pediatric intensive care unit. Intensive Care Med 2007;33:1033-40.

9. Colville G, Pierce C. Children's memories of paediatric intensive care. Arch Dis Child 2005;90(Suppl 2):A35.

10. Playfor S, Thomas D, Choonara I. Recollection of children following intensive care. Arch Dis Child 2000;83:445-8.

11. Rennick JE, Johnston CC, Dougherty G, et al. Children's psychological responses after critical illness and exposure to invasive technology. J Dev Behav Pediatr 2002;23:133-44.

12. Colville $\mathrm{G}$. The impact of admission to paediatric intensive care unit (PICU) on the child and family. In: Ridley S, ed. Critical care focus 12: the psychological challenges of intensive care. London: Blackwell Publishing, 2005:50-61.

13. Milette IH, Carnevale FA. I'm trying to heal...noise levels in a pediatric intensive care unit. Dynamics 2003;14:14-21.

14. Carnevale FA. The experience of critically ill children: narratives of unmaking. Intensive Crit Care Nurs 1997;13:49-52.

15. Colville G, Kerry S, Pierce C. Children's factual and delusional memories of intensive care. Am J Respir Crit Care Med 2008;177:976-82.

16. Muranjan MN, Birajdar SB, Shah HR, et al. Psychological consequences in pediatric intensive care unit survivors: the neglected outcome. Indian Pediatr 2008;45:99-103.

17. Rennick JE, Morin I, Kim D, et al. Identifying children at high risk for psychological sequelae after pediatric intensive care unit hospitalization. Pediatr Crit Care Med 2004;5:358-63.

18. Karande S, Kelkar A, Kulkarni M. Recollections of Indian children after discharge from an intensive care unit. Pediatr Crit Care Med 2005;6:303-7.

19. Rennick JE, McHarg LF, Dell'Api M, et al. Developing the children's critical illness impact scale: capturing stories from children, parents, and staff. Pediatr Crit Care Med 2008;9:252-60.

20. Colville G. The psychologic impact on children of admission to intensive care. Pediatr Clin North Am 2008;55:605-16.

21. Rees G, Gledhill J, Garralda ME, et al. Psychiatric outcome following paediatric intensive care unit (PICU) admission: a cohort study. Intensive Care Med 2004;30:1607-14.

22. Bronner MB, Knoester H, Bos AP, et al. Posttraumatic stress disorder (PTSD) in children after paediatric intensive care treatment compared to children who survived a major fire disaster. Child Adolesc Psychiatry Mental Health 2008;2:9.

23. Board R, Dai JY. Effects of five parent-and-child risk factors on salivary cortisol levels and symptoms of posttraumatic stress disorder in school-age, critically ill children: pilot study. Heart Lung 2010:40:236-46.

24. Colville G, Tighe H, Pierce C. Children's factual and delusional memories of paediatric intensive care: 80. Crit Care Med 2006;34:A20.

25. Judge D, Nadel S, Vergnaud S, et al. Psychiatric adjustment following meningococcal disease treated on a PICU. Intensive Care Med 2002;28:648-50.

26. Vermunt LC, Buysse CM, Joosten KF, et al. Behavioural, emotional, and post-traumatic stress problems in children and adolescents, long term after septic shock caused by Neisseria meningitidis. Br J Clin Psychol 2008;47(Pt 3):251-63.

27. Morrison AL, Gillis J, O'Connell AJ, et al. Quality of life of survivors of pediatric intensive care. Pediatr Crit Care Med 2002;3:1-5.

28. Mestrovic J, Kardum G, Sustic A, et al. Neurodevelopmental disabilities and quality of life after intensive care treatment. $J$ Paediatr Child Health 2007;43:673-76.

29. Knoester H, Bronner MB, Bos AP, et al. Quality of life in children three and nine months after discharge from a paediatric intensive care unit: a prospective cohort study. Health Qual Life Outcomes 2008;6:21.

30. Atkins E, Colville G, John M. A 'biopsychosocial' model for recovery: a grounded theory study of families' journeys after a Paediatric Intensive Care Admission. Intensive Crit Care Nurs 2012:28: 133-40.

31. Piaget J. The language and thought of the child. 3rd edn, London: Routledge, 2001.

32. Jayashree M, Singhi SC, Malhi P. Follow up of survival and quality of life in children after intensive care. Indian Pediatr 2003;40: $303-9$

33. Jones S, Rantell K, Stevens K, et al. Outcome at 6 months after admission for pediatric intensive care: a report of a National Study of Pediatric Intensive Care Units in the United Kingdom. Pediatrics 2006;118:2101-8

34. Small L, Melnyk BM. Early predictors of post-hospital adjustment problems in critically ill young children. Res Nurs Health 2006; 29:622-35. 
35. Taylor A, Butt W, Ciardulli M. The functional outcome and quality of life of children after admission to an intensive care unit. Intensive Care Med 2003;29:795-800.

36. Board R. School-age children's perceptions of their PICU hospitalization. Pediatr Nurs 2005;31:166.

37. Manning JC, Hemingway P, Redsell S. G59(P) long-term psychological and social impact reported by childhood critical illness survivors: a systematic review and thematic synthesis [abstract]. Arch Dis Child 2013:98(Suppl 1):A31.

38. Manning JC, Hemingway P, Redsell SA. Long-term psychosocial impact reported by childhood critical illness survivors: a systematic review. Nurs Crit Care 23 Oct 2013. Epub ahead of print. doi:10.1111/nicc.12049

39. Yin RK. Case study research: design and methods. 4th edn. London: Sage, 2009.

40. Stake RE. The art of case study research. London: Sage, 1995.

41. Crivello G, Camfield L, Woodhead M. How can children tell us about their wellbeing? Exploring the potential of participatory research approaches within young lives. Soc Indicators Res 2009;90:51-72.

42. Paediatric Intensive Care Audit Network. Annual report of the Paediatric Intensive Care Audit network January 2008-December 2010. London, 2011.

43. Emerson RM. Observational field work. Annu Rev Sociol 1981;7: $351-78$

44. World Health Organisation. The international statistical classification of disorders and related health problems (10 revision). 2nd edn. Geneva: WHO, 2005.

45. Salganik MJ, Heckathorn DD. Sampling and estimation in hidden populations using respondent-driven sampling. Sociol Method 2004;34:193-240.

46. Faugier J, Sargeant M. Sampling hard to reach populations. $J A d v$ Nurs 1997;26:790-7.

47. Ritchie J, Lewis J, Elam G. Designing and selecting samples. In Ritchie JLewis J, eds. Qualitative research practice: a guide for social science students and researchers. London: Sage, 2003 $77-108$.

48. Morse JM. Determining sample size. Qual Health Res 2000;10:3-5.

49. White A, Bushin N, Carpena-Mendez F, et al. Using visual methodologies to explore contemporary Irish childhoods. Qual Res 2010;10:143-58.

50. Murphy E, Dingwall R, Greatbatch D, et al. Qualitative research methods in health technology assessment: a review of the literature. Health Technol Assess 1998;2:iii-ix, 1-274

51. Paton J, Crouch W, Camic P. Young offenders' experiences of traumatic life events: a qualitative investigation. Clin Child Psychol Psychiatry 2009;14:43-62.

52. Birbeck D, Drummond M. Interviewing, and listening to the voices of very young children on body image and perceptions of self. Early Child Dev Care 2005;175:579-96.

53. Bagnoli A. Beyond the standard interview: the use of graphic elicitation and arts-based methods. Qual Res 2009;9:547-70.

54. Holliday EL, Harrison LJ, McLeod S. listening to children with communication impairment talking through their drawings. $J$ Early Child Res 2009;7:244-63.

55. Coad J. Using art-based techniques in engaging children and young people in health care consultations and/or research. $J$ Res Nurs 2007;12:487-97.

56. Driessnack M, Furukawa R. Arts-based data collection techniques used in child research. J Spec Pediatr Nurs 2012;17:3-9.

57. Tekola B, Griffin C, Camfield L. Using qualitative methods with poor children in urban ethiopia: opportunities \& challenges. Soc Indicators Res 2009;90:73-87.

58. Gibson F, Mulhall A, Richardson A, et al. A phenomenologic study of fatigue in adolescents receiving treatment for cancer. Oncol Nurs Forum 2005;32:651-60.

59. Smith Percy M. Children from homeless families describe what is special in their lives. Holist Nurs Pract 1995;9:24-33.

60. Herth K. Hope as seen through the eyes of homeless children. J Adv Nurs 1998;28:1053-62.

61. Prosser J. Image based research. London: Falmer Press, 1998.
62. Boyden J, Ennew J. Children in focus: a manual for participatory research with children. Stockholm: Save the Children Sweden, 1997.

63. Leeson C. Asking difficult questions: exploring research methods with children on painful issues. Int J Res Method Educ Published Online: 1 Aug 2013. doi:10.1080/1743727X.2013.820643

64. Kirby P. Involving young researchers: how to enable young people to design and conduct research. York: York Publishing Services, 1999

65. Hill M, Laybourn A, Borland M. Engaging with primary-aged children about their emotions and well-being: methodological considerations. Child Soc 1996;10:129-44.

66. James A, Prout A. Constructing and reconstructing childhood: contemporary issues in the sociological study of childhood. London: Falmer, 1997.

67. Campbell C, Skovdal M, Mupambireyi Z, et al. Exploring children's stigmatisation of AIDS-affected children in Zimbabwe through drawings and stories. Soc Sci Med 2010;71:975-85.

68. Jolley RP, Fenn K, Jones L. The development of children's expressive drawing. Br J Dev Psychol 2004;22:545-67.

69. Bagnoli $A$. Researching identities with multi-method autobiographies Sociol Res 2004;9. http://www.socresonline.org.uk/9/2/bagnoli.html (accessed 20 Dec 20 2012).

70. Banks M. Visual methods in social research. London: Sage, 2001.

71. Harper D. Talking about pictures: a case for photo elicitation. Vis Stud 2002;17:13-26.

72. Hurworth R. Photo-interviewing for research. Soc Res Update (Spring) 2003;40:1-4

73. Truesdell B. Oral history techniques: how to organize and conduct oral history interviews. 2001. http://www.indiana.edu/ cshm/oral history_techniques.pdf (accessed Mar 18 2013).

74. Mays N, Pope C. Qualitative research: rigour and qualitative research. BMJ1995:311:109-12.

75. Seale C. The uality of qualitative research. London: Sage, 1999.

76. Creswell JW. Qualitative inquiry \& research design: choosing among five approaches. London: Sage, 2007.

77. McAdams DP. The stories we live by: personal myths and the making of the self. Guilford Press, 1993.

78. Crossley ML. Introducing narrative psychology: Self, trauma, and the construction of meaning. Buckingham: Open University Press, 2000.

79. McAdams DP. What do we know when we know a person? J Pers 1995;63:365-96.

80. Savory C. Patient and public involvement in translative healthcare research. Clin Governance 2010;15:191-9.

81. INVOLVE. Public involvement in research: impact on ethical aspects of research. Eastleigh: INVOLVE, 2012.

82. INVOLVE. Exploring the impact of public involvement on the quality of research. Eastleigh: INVOLVE, 2013.

83. INVOLVE. Payment for involvement: a guide for making payments to members of the public actively involved in NHS, public health and social care research. Eastleigh: INVOLVE, 2010.

84. Anderson K, Balandin S. The storybook method: research feedback with young participants. Augment Altern Commun 2011;27:279-91.

85. Coyne I, Hayes E, Gallagher P. Research with hospitalized children: ethical, methodological and organizational challenges. Childhood 2009;16:413-29.

86. Baxter R, Long A, Sines D. The legal and ethical status of children in health care in the UK. Nurs Ethics 1998;5:189-99.

87. Alderson P. Ethics. In: Fraser S, Lewis V, Ding S, Kellett M, Robinson C, eds. Doing research with children and young people. London: Sage, 2004:97-112.

88. Clavering EK, McLaughlin J. Children's participation in health research: from objects to agents? Child Care Health Dev 2010;36:603-11.

89. Faux SA, Walsh M, Deatrick JA. Intensive interviewing with children and adolescents. West J Nurs Res 1988;10:180-94.

90. Lansdown $\mathrm{G}$. The evolving capacities of the child. Florence, Italy: UNICEF Innocenti Research Centre, 2005

91. Nursing and Midwifery Council. The code: standards of conduct, performance and ethics for nurses and midwives. London: NMC, 2008.

92. Medical Research Council. Developing and evaluating complex interventions: new guidance. London: MRC, 2008 